\title{
Preparation of oxo-substituted $\alpha$-chloro ethers and their reaction with samarium diiodide
}

\author{
Tore Skjæret and Tore Benneche* \\ Department of Chemistry, University of Oslo, PO Box 1033, Blindern, 0315 Oslo, Norway \\ E-mail: tore.benneche@kjemi.uio.no
}

(received 23 Jun 01; accepted 18 Oct 01; published on the web 26 Oct 01)

\begin{abstract}
Oxo-substituted $\alpha$-chloro ethers have been prepared by cleavage of the corresponding $O, S$ acetals with sulfuryl dichloride. Some of these $\alpha$-chloro ethers give hydroxysubstituted oxygen heterocycles when reacted with samarium diiodide.
\end{abstract}

Keywords: $\alpha$-Chloro ethers, samarium diiodide, hydroxysubstituted oxygen heterocycles, intramolecular samarium Barbier reaction

\section{Introduction}

Oxo-substituted $\alpha$-chloro ethers like 1 (Fig. 1) are hardly known in the literature. One of the few examples is the fluorinated $\alpha$-chloro ether 2 , which was prepared by chlorination of a methoxy group. ${ }^{1}$<smiles>CC(=O)C(C)OCCl</smiles>

1<smiles>O=C(C=CC(F)(F)F)C(F)(OCCl)C(F)(F)F</smiles>

2

$$
\mathrm{n}=1,2,3 \ldots
$$

\section{Figure 1}

We have previously prepared a number of $\alpha$-chloro ethers by cleavage of $O, S$-acetals with sulfuryl dichloride. ${ }^{2}$ (Scheme 1) 


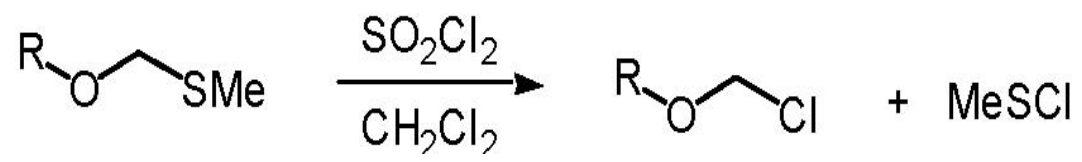

\section{Scheme 1}

This method has also been used to prepare 4-acetyl- chloromethoxybenzene. ${ }^{2 a}$ We believed that the method could be useful in the synthesis of $\alpha$-chloro ethers like $\mathbf{1}$, since the requested $O, S$-acetals could in principle be accessible from readily available hydroxy ketones. $\alpha$-Halo ethers react with samarium diiodide in the presence of ketones to give $\alpha$-alkoxy alcohols. ${ }^{3}$ To our knowledge an intramolecular version of this reaction has not been documented. Such a reaction would be an example of an intramolecular Samarium-Barbier reaction and would give hydroxysubstituted oxy-rings. The ring size being dependent on the chain lenght between the $\alpha$ chloro ether function and the oxo-group. A number of oxygen heterocyles are important compounds; oxolanes and oxanes for instance occur in a wide variety of biologically active compounds. ${ }^{4}$ In this paper we present our results in the preparation of oxy-substituted $\alpha$-chloro ethers and in the investigation of the intramolecular Samarium-Barbier reaction of oxysubstituted $\alpha$-chloro ethers.

\section{Results and Discussion}

A convenient way to make $O, S$-acetals from alcohols, is to react the alcohol with chloromethyl methyl sulfide under basic conditions. ${ }^{5}$ This method (Method A, Table 1) proved useful for the hydroxy ketones 3d, 3f and $\mathbf{3 g}$ (Table 1, entries 4, 6 and 7). In the case of 3a, 3b, 3c and 3e, however, the method was useless (entries 1, 2, 3 and 5).

$O, S$-acetals can also be formed from alcohols by a reacting the alcohol with DMSO in the presence of acetic anhydride and acetic acid. ${ }^{6}$ This protocol (Method B, Table 1) gave mostly good yields where Method A had failed (entries 2 - 5). 
Table 1. Preparation of hemithioacetals

Entry

${ }^{\mathrm{a}}$ Method A: ${ }^{5} \mathrm{ClCH}_{2} \mathrm{Me}, \mathrm{NaH}, \mathrm{NaI} / \mathrm{DMF} .{ }^{\mathrm{b}}$ Method B: ${ }^{6} \mathrm{DMSO} / \mathrm{Ac} 2 \mathrm{O} / \mathrm{Ac} \mathrm{OH} .{ }^{\mathrm{c}}$ Isolated

Cleavage of the $O, S$-acetals 4 with sulfuryl dichloride gave the corresponding $\alpha$-chloro ethers 5 in almost quantitative yield (Table 2, entries 1-5 and 7) except in one case (4f, entry 6) where a mixture of products was obtained. 
Table 2 Preparation of $\alpha$-chloro ethers

$$
\underset{4}{\mathrm{R}-\mathrm{OCH}_{2} \mathrm{SMe}} \stackrel{\mathrm{SO}_{2} \mathrm{Cl}_{2}}{\longrightarrow} \underset{5}{\mathrm{R}}-\mathrm{OCH}_{2} \mathrm{Cl}+\mathrm{MeSCl}
$$

Entry Compound

${ }^{a}$ Crude product. ${ }^{b}$ H-NMR signal of the crude product indicated the presence of an $\alpha$-chloro ether

The reason why the $O, S$-acetal 4 f does not cleanly give the corresponding $\alpha$-chloro ether, must come from the position of the acetyl group and the fact that the acetyl group can enolize. This because the para-isomer 4-acetyl-chloromethoxybenzene has been synthesized in good yield by cleavage of an $O, S$-acetal with sulfuryl dichloride ${ }^{2 a}$ and the $O, S$-acetal $\mathbf{4 g}$, which does not have an enolizable keto group, is cleanly cleaved to the corresponding $\alpha$-chloro ether. 2Formyl-chloromethoxybenzene has also been prepared in good yield by cleavage of the corresponding $O, S$-acetal with sulfuryl dichloride. ${ }^{8}$ 
The cyclization of the $\alpha$-chloro ether 5a with samarium diiodide met with little success. No oxetane formation was observed (Table 3, entry 1). This was not surprising since formation of a 4-membered ring from a simple halide in a similar reaction gave only $5 \%$ yield. ${ }^{9}$ The formation five membered rings were easier and the 3hydroxyoxolanes $\mathbf{6 b}$ and $\mathbf{6 c}$ were obtained in moderate yields when the corresponding $\alpha$-chloro ethers were treated with two equivalents of samarium diiodide in THF at $-78{ }^{\circ} \mathrm{C}$ (Table 3 , entries 2 and 3). The diastereoselectivity in the cyclization of 5c was $2.5: 1$. The phenyl ketone 5e gave, however, no oxolane (entry 5). The 6-membered ring $\mathbf{6 d}$ was formed in $47 \%$ yield (entry 4 ).

Table 3. Preparation of 3-hydroxyoxolanes and 3-hydroxy-3-methyloxane

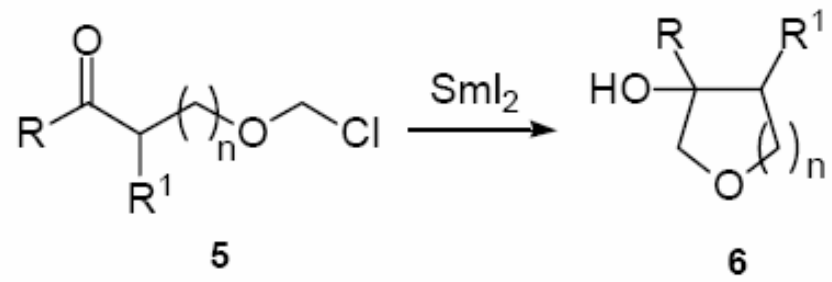

\begin{tabular}{llllll}
\hline Entry & Compound & $\mathrm{n}$ & $\mathrm{R}$ & $\mathrm{R} 1$ & Yield of $\mathbf{6} a$ \\
\hline 1 & $\mathbf{5 a}$ & 0 & $\mathrm{Me}$ & $\mathrm{H}$ & 0 \\
2 & $\mathbf{5 b}$ & 1 & $\mathrm{Me}$ & $\mathrm{H}$ & 54 \\
3 & $\mathbf{5 c}$ & 1 & $\mathrm{Me}$ & $\mathrm{Me}$ & 65 \\
4 & $\mathbf{5 d}$ & 2 & $\mathrm{Me}$ & $\mathrm{H}$ & 47 \\
5 & $\mathbf{5 e}$ & 1 & $\mathrm{Ph}$ & $\mathrm{H}$ & 0 \\
\hline${ }^{\mathrm{a}}$ Isolated & & & &
\end{tabular}

When the $\alpha$-chloro ether $\mathbf{5 g}$ was treated with samarium diiodide the oxepinon 7 was formed together with the oxolane $\mathbf{6 g}$ (Scheme 2). The oxepinon was the main product.

The oxepinon can be formed by hydride elimination from compound $\mathbf{8}$, which may be formed by a nucleophilic attac by an organosamarium species ortho to the keto group. This would resemble an 1,4-addition by a nucleophile to an $\alpha, \beta$-unsaturated ketone.

In summary oxy-substituted $\alpha$-chloro ethers are readily made by cleavage of $O, S$-acetals by sulfuryl dichloride. $\alpha$-Chloro ethers are readily reduced by samarium diiodide and oxosubstituted $\alpha$-chloro ethers may be cyclized to five or six membered rings by samarium diiodide. 


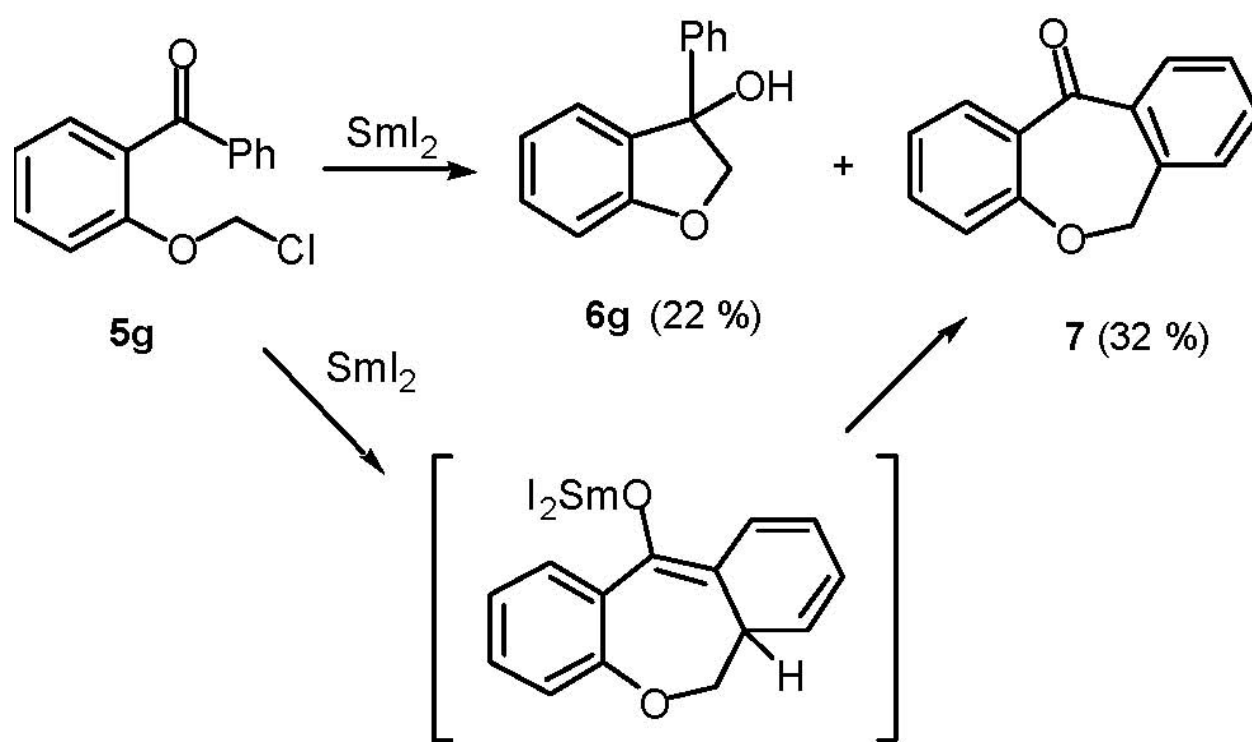

8

Scheme 2

\section{Experimental Section}

General Procedures. All reactions were conducted under an inert amosphere of either $\operatorname{Ar}$ or $\mathrm{N}_{2}$. THF was distilled from sodium and benzophenone. Dichloromethane, acetonitrile and $N, N$ dimethylformamide (DMF) were dried with $\mathrm{CaH}_{2}$ before distillation. NMR spectra were recorded at $300 \mathrm{MHz}\left({ }^{1} \mathrm{H}\right)$ and at $75 \mathrm{MHz}\left({ }^{13} \mathrm{C}\right)$ on a Bruker Avance DPX 300 instrument. Mass spectra, under electron impact conditions, were recorded at $70 \mathrm{eV}$ ionizing energy on a Fision ProSpec instrument. The spectra are presented as $m / z$ ( $\%$ rel. int.). The melting points are uncorrected.

General procedures for the preparation of the $\boldsymbol{O}, \boldsymbol{S}$-acetals (4). Method A: $:^{5}$ The alcohol 3 $(10.0 \mathrm{mmol})$ in DMF $(20 \mathrm{~mL})$ was added to a mixture of sodium hydride $(0.38 \mathrm{~g} 15.0 \mathrm{mmol}, 95$ $\%$ in paraffine $)$ and $\mathrm{NaI}(1.5 \mathrm{~g}, 10 \mathrm{mmol})$ at $0{ }^{\circ} \mathrm{C}$ under $\mathrm{N}_{2}$. After stirring for 15 min was chloromethyl methyl sulfide $(1.10 \mathrm{~mL}, 13.0 \mathrm{mmol})$ added dropwise. The mixture was stirred for $10 \mathrm{~h}$ while reaching ambient temperature before ice/water was added, the product extracted into diethyl ether, dried $\left(\mathrm{MgSO}_{4}\right)$ and the solvent removed under reduced pressure. The crude product was purified by distillation, recrystallization or flash chromatography on silica gel.

2-Acetyl-1-methylthiomethoxybenzene (4f). Eluent hexane-ethyl acetate $10: 1 ; \mathrm{mp} 33.5$ -

$34.5{ }^{\circ} \mathrm{C}$ (hexane); (Found: $\mathrm{M}^{+}, 196.0552 . \mathrm{C}_{10} \mathrm{H}_{12} \mathrm{O}_{2} \mathrm{~S}$ requires 196.0558); ${ }^{1} \mathrm{H}-\mathrm{NMR} \delta(300 \mathrm{MHz}$, $\left.\mathrm{CDCl}_{3}\right) 2.25\left(3 \mathrm{H}, \mathrm{s}, \mathrm{SCH}_{3}\right), 2.62\left(3 \mathrm{H}, \mathrm{s}, \mathrm{CH}_{3}\right), 5.22\left(2 \mathrm{H}, \mathrm{s}, \mathrm{CH}_{2}\right), 6.96-7.05(2 \mathrm{H}, \mathrm{m}, \mathrm{Ar}),, 7.40-$ $7.43(1 \mathrm{H}, \mathrm{m}, \mathrm{Ar}), 7.69-7.72(1 \mathrm{H}, \mathrm{m}, \mathrm{Ar}) ;{ }^{13} \mathrm{C}-\mathrm{NMR} \delta\left(75 \mathrm{MHz}, \mathrm{CDCl}_{3}\right) 15.1\left(\mathrm{SCH}_{3}\right), 31.7\left(\mathrm{CH}_{3}\right)$, $72.9\left(\mathrm{CH}_{2}\right), 113.8(\mathrm{CH}), 121.5(\mathrm{CH}), 129.5(\mathrm{C}), 130.5(\mathrm{CH}), 133.2(\mathrm{CH}), 156.2(\mathrm{C}), 199.6(\mathrm{CO})$; 
$\mathrm{m} / \mathrm{z}$ (EI) $196\left(\mathrm{M}^{+}, 7 \%\right), 149$ (20), 61 (100).

2-Methylthiomethoxybenzophenone (4g). Mp. $65-66{ }^{\circ} \mathrm{C}$ (hexane) (Found: $\mathrm{M}^{+}, 258.0709$. $\mathrm{C}_{15} \mathrm{H}_{14} \mathrm{O}_{2} \mathrm{~S}$ requires 258.0715); ${ }^{1} \mathrm{H}-\mathrm{NMR} \delta\left(300 \mathrm{MHz}, \mathrm{CDCl}_{3}\right) 1.90\left(3 \mathrm{H}, \mathrm{s}, \mathrm{SCH}_{3}\right), 5.02(2 \mathrm{H}, \mathrm{s}$, $\left.\mathrm{CH}_{2}\right), 7.01-7.07(2 \mathrm{H}, \mathrm{m}, \mathrm{Ar}), 7.34-7.52(5 \mathrm{H}, \mathrm{m}, \mathrm{Ar}), 7.79-7.81(1 \mathrm{H}, \mathrm{m}, \mathrm{Ar}) ;{ }^{13} \mathrm{CNMR} \delta(75 \mathrm{MHz}$, $\left.\mathrm{CDCl}_{3}\right) 14.3\left(\mathrm{SCH}_{3}\right), 72.7\left(\mathrm{CH}_{2}\right), 114.2(\mathrm{CH}), 121.5(\mathrm{CH}), 128.2(\mathrm{CH}), 129.5(\mathrm{CH}), 129.7(\mathrm{CH})$, $130.3(\mathrm{C}), 131.4(\mathrm{CH}), 132.9(\mathrm{CH}), 137.7(\mathrm{C}), 154.3(\mathrm{C}), 196,1(\mathrm{CO}) ; \mathrm{m} / \mathrm{z}(\mathrm{EI}) 258\left(\mathrm{M}^{+}, 6 \%\right.$ \%), 194 (6), 211 (21), 182 (6), 137 (8), 121 (10), 77 (18), 61 (100).

Method B: ${ }^{6}$ A mixture of the alcohol $3(10.0 \mathrm{mmol})$, DMSO $(33 \mathrm{~mL})$, acetic acid $(6.5 \mathrm{~mL})$, acetic anhydride $(33 \mathrm{~mL})$ and water $(0.7 \mathrm{~mL})$ was stirred for $48 \mathrm{~h}$ at ambient temperature before it was poured into a cold solution of $10 \% \mathrm{Na}_{2} \mathrm{CO}_{3}$ in water and extracted with chloroform. The organic phase was washed with water $(5 \mathrm{x})$, dried $\left(\mathrm{MgSO}_{4}\right)$ and evaporated. The crude product was purified by flash chromatography on silica gel.

1-Methylthiomethoxy-2-propanone (4a). Eluent hexane-ethyl acetate 5:1 (Found: $\mathrm{M}^{+}$, 134.0394. $\mathrm{C}_{5} \mathrm{H}_{10} \mathrm{O}_{2} \mathrm{~S}$ requires 134.0402); ${ }^{1} \mathrm{H}-\mathrm{NMR} \delta\left(300 \mathrm{MHz}, \mathrm{CDCl}_{3}\right) 2.04\left(3 \mathrm{H}, \mathrm{s}, \mathrm{CH}_{3}\right), 2.05$ $\left(3 \mathrm{H}, \mathrm{s}, \mathrm{CH}_{3}\right), 4.08\left(2 \mathrm{H}, \mathrm{s}, \mathrm{CH}_{2}\right), 4.61\left(2 \mathrm{H}, \mathrm{s}, \mathrm{CH}_{2}\right) ;{ }^{13} \mathrm{C}-\mathrm{NMR} \delta\left(75 \mathrm{MHz}, \mathrm{CDCl}_{3}\right) 13.7\left(\mathrm{CH}_{3} \mathrm{~S}\right)$, $26.2\left(\mathrm{CH}_{3}\right), 72.2\left(\mathrm{CH}_{2}\right), 75.3\left(\mathrm{CH}_{2}\right), 205.3(\mathrm{C}) ; \mathrm{m} / \mathrm{z}(\mathrm{EI}) 134\left(\mathrm{M}^{+}, 0.7 \%\right), 87(29), 61(100), 58$ (45), 57 (25), 43 (40).

4-Methylthiomethoxy-2-butanone (4b). Eluent hexane-ethyl acetate 5:1 (Found: $\mathrm{M}^{+}, 148.0562$. $\mathrm{C}_{6} \mathrm{H}_{12} \mathrm{O}_{2} \mathrm{~S}$ requires 148.0558); $\delta \mathrm{H}\left(300 \mathrm{MHz}, \mathrm{CDCl}_{3}\right) 2.03\left(3 \mathrm{H}, \mathrm{s}, \mathrm{CH}_{3} \mathrm{~S}\right), 2.09\left(3 \mathrm{H}, \mathrm{s}, \mathrm{CH}_{3} \mathrm{~S}\right)$, $2.60\left(2 \mathrm{H}, \mathrm{t}, \mathrm{CH}_{2}, J\right.$ 6.1), $3.68\left(2 \mathrm{H}, \mathrm{t}, \mathrm{CH}_{2}, J\right.$ 6.1), $4.52\left(2 \mathrm{H}, \mathrm{s}, \mathrm{CH}_{2}\right) ; \delta \mathrm{C}\left(75 \mathrm{MHz}, \mathrm{CDCl}_{3}\right) 13.7$ $\left(\mathrm{CH}_{3} \mathrm{~S}\right), 30.1\left(\mathrm{CH}_{3}\right), 43.1\left(\mathrm{CH}_{2}\right), 62.8\left(\mathrm{CH}_{2}\right), 75.2\left(\mathrm{CH}_{2}\right), 206.5(\mathrm{C}) ; \mathrm{m} / \mathrm{z}(\mathrm{EI}) 148\left(\mathrm{M}^{+}, 2 \%\right), 101$ (59), 71 (27), 61 (22), 43 (100).

4-Methylthiomethoxy-3-methyl-2-butanone (4c). Eluent hexane-ethyl acetate 12:1 (Found: $\mathrm{M}^{+}, 162.0720 . \mathrm{C}_{7} \mathrm{H}_{14} \mathrm{O}_{2} \mathrm{~S}$ requires 162.0715); ${ }^{1} \mathrm{H}-\mathrm{NMR} \delta\left(300 \mathrm{MHz}, \mathrm{CDCl}_{3}\right) 1.03\left(3 \mathrm{H}, \mathrm{d}, \mathrm{CH}_{3}, J\right.$ 7.1), $2.04\left(3 \mathrm{H}, \mathrm{s}, \mathrm{SCH}_{3}\right), 2.11\left(3 \mathrm{H}, \mathrm{s}, \mathrm{CH}_{3}\right), 2.75(1 \mathrm{H}, \mathrm{ddq}, \mathrm{CH}, J$ 7.5, 7.1 and 5.3), $3.50(1 \mathrm{H}$, dd, $\mathrm{CH}, J 9.3$ and 5.3), $3.60(1 \mathrm{H}$, dd, $\mathrm{CH}, J 9.3$ and 7.5$), 4.52\left(2 \mathrm{H}, \mathrm{s}, \mathrm{OCH}_{2} \mathrm{~S}\right) ;{ }^{13} \mathrm{C}-\mathrm{NMR} \delta(75 \mathrm{MHz}$, $\left.\mathrm{CDCl}_{3}\right) 13.2\left(\mathrm{CH}_{3}\right), 13.8\left(\mathrm{CH}_{3}\right), 28.6\left(\mathrm{CH}_{3}\right), 46.7(\mathrm{CH}), 69.5\left(\mathrm{CH}_{2}\right), 75.3\left(\mathrm{CH}_{2}\right), 210.4(\mathrm{CO}) ; \mathrm{m} / \mathrm{z}$ (EI) $162\left(\mathrm{M}^{+}, 0.5 \%\right), 115(55), 85(23), 61(30), 43$ (100).

5-Methylthiomethoxy-2-pentanone (4d). Eluent hexane-ethyl acetate 5:1 (Found: $\mathrm{M}^{+}$, 162.0726. $\mathrm{C}_{7} \mathrm{H}_{14} \mathrm{O}_{2} \mathrm{~S}$ requires 162.0715); ${ }^{1} \mathrm{H}-\mathrm{NMR} \delta\left(300 \mathrm{MHz}, \mathrm{CDCl}_{3}\right) 1.75\left(2 \mathrm{H}, \mathrm{tt}, \mathrm{CH}_{2}, J 6.2\right.$ and 7.2), $2.03\left(3 \mathrm{H}, \mathrm{s}, \mathrm{CH}_{3}\right), 2.04\left(3 \mathrm{H}, \mathrm{s}, \mathrm{CH}_{3}\right), 2.43\left(2 \mathrm{H}, \mathrm{t}, \mathrm{CH}_{2}, J\right.$ 7.2), $3.41\left(2 \mathrm{H}, \mathrm{t}, \mathrm{CH}_{2}, J 6.2\right)$, $4.49\left(2 \mathrm{H}, \mathrm{s}, \mathrm{OCH}_{2} \mathrm{~S}\right) ;{ }^{13} \mathrm{C}-\mathrm{NMR} \delta\left(75 \mathrm{MHz}, \mathrm{CDCl}_{3}\right) 13.8\left(\mathrm{CH}_{3} \mathrm{~S}\right), 23.3\left(\mathrm{CH}_{3}\right), 29.7\left(\mathrm{CH}_{2}\right), 40.1$ $\left(\mathrm{CH}_{2}\right), 66.9\left(\mathrm{CH}_{2}\right), 75.0\left(\mathrm{CH}_{2}\right), 208.0(\mathrm{CO}) ; \mathrm{m} / \mathrm{z}(\mathrm{EI}) 162\left(\mathrm{M}^{+}, 0.2 \%\right), 115(69), 101$ (15), 85 (100), 43 (29).

3-Methylthiomethoxy-1-phenyl-1-propanone (4e). Eluent hexane-ethyl acetate 9:1 (Found: $\mathrm{M}^{+}-\mathrm{SCH}_{3}, 163.0764 . \mathrm{C}_{10} \mathrm{H}_{11} \mathrm{O}_{2}$ requires 163.0759); ' $\mathrm{H}-\mathrm{NMR} \delta\left(300 \mathrm{MHz}, \mathrm{CDCl}_{3}\right) 2.09(3 \mathrm{H}, \mathrm{s}$, $\left.\mathrm{SCH}_{3}\right), 3.23\left(2 \mathrm{H}, \mathrm{t}, \mathrm{CH}_{2}, J 6.3\right), 3.94\left(2 \mathrm{H}, \mathrm{t}, \mathrm{CH}_{2}, J 6.3\right), 4.62\left(2 \mathrm{H}, \mathrm{s}, \mathrm{OCH}_{2} \mathrm{~S}\right), 7,40$ - $7.56(3 \mathrm{H}$, m, Ar), 7.91 - $7.94(2 \mathrm{H}, \mathrm{m}, \mathrm{Ar}) ;{ }^{13} \mathrm{C}-\mathrm{NMR} \delta\left(75 \mathrm{MHz}, \mathrm{CDCl}_{3}\right) 13.8\left(\mathrm{SCH}_{3}\right), 38,3\left(\mathrm{CH}_{2}\right), 63.3$ $\left(\mathrm{CH}_{2}\right), 75.4\left(\mathrm{OCH}_{2} \mathrm{~S}\right), 128.0(2 \times \mathrm{CH}), 128.5(2 \times \mathrm{CH}), 133.1(\mathrm{CH}), 136.8(\mathrm{C}) 197.9(\mathrm{CO})$. 
General procedure for the synthesis of the $\mathbb{Q}$-chloro ethers (5). Sulfuryl dichloride $(0.16 \mathrm{~mL}$, $2.0 \mathrm{mmol})$ in dichloromethane $(4 \mathrm{~mL})$ was added to a solution of the $O, S$-acetal $4(2.0 \mathrm{mmol})$ in dichloromethane $(6 \mathrm{~mL})$ at $0{ }^{\circ} \mathrm{C}$ under $\mathrm{N}_{2}$. The mixture was stirred for $10 \mathrm{~min}$ before it was evaporated under reduced pressure. The crude product could be used in the next step without further purification. Note: The $\alpha$-chloro ethers $\mathbf{5}$ are reactive compounds and thus potentially toxic. Care should be taken to avoid exposure.

1-Chloromethoxy-2-propanone (5a). ${ }^{1} \mathrm{H}-\mathrm{NMR} \delta\left(300 \mathrm{MHz}, \mathrm{CDCl}_{3}\right) 2.13\left(3 \mathrm{H}, \mathrm{s}, \mathrm{CH}_{3}\right), 4.24$ $\left(2 \mathrm{H}, \mathrm{s}, \mathrm{CH}_{2}\right), 5.47\left(2 \mathrm{H}, \mathrm{s}, \mathrm{CH}_{2}\right) ;{ }^{13} \mathrm{C}-\mathrm{NMR} \delta\left(75 \mathrm{MHz}, \mathrm{CDCl}_{3}\right) 26.4\left(\mathrm{CH}_{3} \mathrm{~S}\right), 73.7\left(\mathrm{CH}_{2}\right), 81.9$ $\left(\mathrm{CH}_{2}\right), 203.8(\mathrm{C})$.

4-Chloromethoxy-2-butanone (5b). ${ }^{1} \mathrm{H}-\mathrm{NMR} \delta\left(300 \mathrm{MHz}, \mathrm{CDCl}_{3}\right) 2.13\left(3 \mathrm{H}, \mathrm{s}, \mathrm{CH}_{3}\right), 2.69(2 \mathrm{H}$, t, $\mathrm{CH}_{2}, J$ 6.1), $3.89\left(2 \mathrm{H}, \mathrm{t}, \mathrm{CH}_{2}, J 6.1\right), 5.42\left(2 \mathrm{H}, \mathrm{s}, \mathrm{CH}_{2}\right) ;{ }^{13} \mathrm{C}-\mathrm{NMR} \delta\left(75 \mathrm{MHz}, \mathrm{CDCl}_{3}\right) 30.2$ $\left(\mathrm{CH}_{3} \mathrm{~S}\right), 42.6\left(\mathrm{CH}_{2}\right), 65.2\left(\mathrm{CH}_{2}\right), 82.9\left(\mathrm{CH}_{2}\right), 205.9(\mathrm{C})$.

4-Chloromethoxy-3-methyl-2-butanone (5c). ${ }^{1} \mathrm{H}-\mathrm{NMR} \delta\left(300 \mathrm{MHz}, \mathrm{CDCl}_{3}\right) 1.03\left(3 \mathrm{H}, \mathrm{d}, \mathrm{CH}_{3}\right.$, $J$ 7.2), $2.10\left(3 \mathrm{H}, \mathrm{s}, \mathrm{CH}_{3}\right), 2.76(1 \mathrm{H}$, ddq, $\mathrm{CH}, J$ 7.5, 7.2 and 5.4), $3.65(1 \mathrm{H}$, dd, $\mathrm{CH}, J 9.5$ and 5.4), $3.73\left(1 \mathrm{H}, \mathrm{dd}, \mathrm{CH}, J 9.5\right.$ and 7.5), $5.35(1 \mathrm{H}, \mathrm{d}, \mathrm{CH}, J 5.5), 5.39(1 \mathrm{H}, \mathrm{d}, \mathrm{CH}, J 5.5) ;{ }^{13} \mathrm{C}-\mathrm{NMR}$ $\delta\left(75 \mathrm{MHz}, \mathrm{CDCl}_{3}\right) 13.1\left(\mathrm{CH}_{3}\right), 28.6\left(\mathrm{CH}_{3}\right), 46.2(\mathrm{CH}), 71.5\left(\mathrm{CH}_{2}\right), 82.8\left(\mathrm{CH}_{2}\right), 209.7(\mathrm{C})$.

5-Chloromethoxy-2-pentanone (5d). ${ }^{1} \mathrm{H}-\mathrm{NMR} \delta\left(300 \mathrm{MHz}, \mathrm{CDCl}_{3}\right) 1.79(1 \mathrm{H}, \mathrm{tt}, \mathrm{CH}, J 7.1$ and 6.1), $2.05\left(3 \mathrm{H}, \mathrm{s}, \mathrm{CH}_{3}\right), 2.45\left(2 \mathrm{H}, \mathrm{d}, \mathrm{CH}_{2}, J 7.1\right), 3.60\left(2 \mathrm{H}, \mathrm{d}, \mathrm{CH}_{2}, J 6.1\right), 5.38\left(2 \mathrm{H}, \mathrm{s}, \mathrm{CH}_{2}\right) ;{ }^{13} \mathrm{C}-$ NMR $\delta\left(75 \mathrm{MHz}, \mathrm{CDCl}_{3}\right) 22.8\left(\mathrm{CH}_{3}\right), 29.8\left(\mathrm{CH}_{2}\right), 39.5\left(\mathrm{CH}_{2}\right), 69.3\left(\mathrm{CH}_{2}\right), 82.9\left(\mathrm{CH}_{2}\right), 207.7$ (C).

3-Chloromethoxy-1-phenyl-1-propanone (5e). ${ }^{1} \mathrm{H}-\mathrm{NMR} \delta\left(300 \mathrm{MHz}, \mathrm{CDCl}_{3}\right) 3.29(2 \mathrm{H}, \mathrm{t}$, $\mathrm{CH}_{2}, J$ 6.3), 4,13 (2H, t, $\mathrm{CH}_{2}, J$ 6.3), $5.53\left(2 \mathrm{H}, \mathrm{s}, \mathrm{OCH}_{2} \mathrm{Cl}\right), 7,44$ - 7.58 (3H, m, Ar), 7.94 - 7.97 $(2 \mathrm{H}, \mathrm{m}, \mathrm{Ar}) ;{ }^{13} \mathrm{C}-\mathrm{NMR} \delta\left(75 \mathrm{MHz}, \mathrm{CDCl}_{3}\right) 37,8\left(\mathrm{CH}_{2}\right), 65.8\left(\mathrm{CH}_{2}\right), 83.2\left(\mathrm{CH}_{2}\right), 128.0(2 \times \mathrm{CH})$, $128.7(2 \times \mathrm{CH}), 133.3(\mathrm{CH}), 136.8(\mathrm{C}) 197.4(\mathrm{CO})$.

2-Chloromethoxybenzophenone (5g). ${ }^{1} \mathrm{H}-\mathrm{NMR} \delta\left(300 \mathrm{MHz}, \mathrm{CDCl}_{3}\right) 5.73\left(2 \mathrm{H}, \mathrm{s}, \mathrm{CH}_{2}\right), 7.16$ $7.80(9 \mathrm{H}, \mathrm{m}, \mathrm{Ar}) ;{ }^{13} \mathrm{C}-\mathrm{NMR} \delta\left(75 \mathrm{MHz}, \mathrm{CDCl}_{3}\right) 76.8\left(\mathrm{CH}_{2}\right), 114.5(\mathrm{CH}), 123.2(\mathrm{CH}), 129.0(2 \mathrm{x}$ $\mathrm{CH}), 129.7(2 \times C H), 129.8(\mathrm{CH}), 130.3(\mathrm{C}), 131.7(\mathrm{CH}), 133.2(\mathrm{CH}), 137.2(\mathrm{C}), 152.9(\mathrm{C}), 195.3$ (C).

General procedure for the synthesis of 3-hydroxyoxolanes and 3-hydroxy-3-methyloxane. The $\alpha$-chloro ether $5(2.0 \mathrm{mmol})$ in dry THF $(4 \mathrm{~mL})$ was added to a solution of $\mathrm{SmI}_{2}(4.0 \mathrm{mmol})$ in THF $(10 \mathrm{~mL})$ at $-78{ }^{\circ} \mathrm{C}$ under $\mathrm{N}_{2}$. The mixture was stirred for $6 \mathrm{~h}$ while slowly reaching ambient temperature before it was poured into a saturated solution of $\mathrm{K}_{2} \mathrm{CO}_{3}$. The product was extracted into diethyl ether and the organic phase washed with a saturated solution of $\mathrm{K}_{2} \mathrm{CO}_{3}$ and a saturated solution of $\mathrm{NaCl}$ before it was dried $\left(\mathrm{MgSO}_{4}\right)$ and evaporated. The crude product was purified by flash chromatography on silica gel.

3-Hydroxy-3-methyloxolane (6b). ${ }^{10}$ Eluent hexane-ethyl acetate 1:2. (Found: $\mathrm{M}^{+}, 102.0690$. $\mathrm{C}_{5} \mathrm{H}_{10} \mathrm{O}_{2}$ requires 102.0681); ${ }^{1} \mathrm{H}-\mathrm{NMR} \delta\left(300 \mathrm{MHz}, \mathrm{CDCl}_{3}\right) 1.36\left(3 \mathrm{H}, \mathrm{s}, \mathrm{CH}_{3}\right), 1.85-1.93(2 \mathrm{H}$, m, $\left.\mathrm{CH}_{2}\right), 2.55(1 \mathrm{H}, \mathrm{s}, \mathrm{OH}), 3.47\left(2 \mathrm{H}, \mathrm{d}, \mathrm{CH}_{2}, J\right.$ 9.1), $3.64\left(2 \mathrm{H}, \mathrm{d}, \mathrm{CH}_{2}, J\right.$ 9.1), 3.83 - 3.87 (1H, m, 
$\left.\mathrm{CH}_{2}\right), 3.94-3.99\left(1 \mathrm{H}, \mathrm{m}, \mathrm{CH}_{2}\right) ;{ }^{13} \mathrm{C}-\mathrm{NMR} \delta\left(75 \mathrm{MHz}, \mathrm{CDCl}_{3}\right) 24.0\left(\mathrm{CH}_{3}\right), 41.0\left(\mathrm{CH}_{2}\right), 67.6$ $\left(\mathrm{CH}_{2}\right), 78.0(\mathrm{CH}), 79.4\left(\mathrm{CH}_{2}\right) ; \mathrm{m} / \mathrm{z}(\mathrm{EI}) 102\left(\mathrm{M}^{+}, 100 \%\right), 87$ (56), 72 (76), 57 (22).

3-Hydroxy-3,4-dimethyloxolane (6c). Eluent hexane-ethyl acetate 1:2. Diastereomer 1: (Found: $\mathrm{M}^{+}, 116.0829 . \mathrm{C}_{6} \mathrm{H}_{12} \mathrm{O}_{2}$ requires 116.0837$) ;{ }^{1} \mathrm{H}-\mathrm{NMR} \delta\left(300 \mathrm{MHz}, \mathrm{CDCl}_{3}\right) 0.91\left(3 \mathrm{H}, \mathrm{d}, \mathrm{CH}_{3}, J\right.$ 6.8), $1.22\left(3 \mathrm{H}, \mathrm{s}, \mathrm{CH}_{3}\right), 1.96(1 \mathrm{H}, \mathrm{ddq}, \mathrm{CH}, J 10.2,8.1$ and 6.8$), 2.18(1 \mathrm{H}, \mathrm{s}, \mathrm{OH}), 3.47(1 \mathrm{H}, \mathrm{dd}$, $\mathrm{CH}, J 10.2$ and 8.1), $3.60(1 \mathrm{H}, \mathrm{d}, \mathrm{CH}, J 9.2), 3.70(1 \mathrm{H}, \mathrm{d}, \mathrm{CH}, J 9.2), 3.70(1 \mathrm{H}, \mathrm{dd}, \mathrm{CH}, J 8.1$ and 8.1); ${ }^{13} \mathrm{C}-\mathrm{NMR} \delta\left(75 \mathrm{MHz}, \mathrm{CDCl}_{3}\right) 8.8\left(\mathrm{CH}_{3}\right), 21.9\left(\mathrm{CH}_{3}\right), 43.1(\mathrm{CH}), 73.9\left(\mathrm{CH}_{2}\right), 78.0(\mathrm{C}), 80.1$ $\left(\mathrm{CH}_{2}\right)$. Diastereomer 2: (Found: $\mathrm{M}^{+}, 116.0838 . \mathrm{C}_{6} \mathrm{H}_{12} \mathrm{O}_{2}$ requires 116.0837); ${ }^{1} \mathrm{H}-\mathrm{NMR} \delta(300$ $\left.\mathrm{MHz}, \mathrm{CDCl}_{3}\right) 0.93\left(3 \mathrm{H}, \mathrm{d}, \mathrm{CH}_{3}, J\right.$ 7.2), $1.23\left(3 \mathrm{H}, \mathrm{s}, \mathrm{CH}_{3}\right), 2.01(1 \mathrm{H}, \mathrm{s}, \mathrm{OH}), 2.10(1 \mathrm{H}, \mathrm{ddq}, \mathrm{CH}, J$ 8.5, 7.2 and 5.4), $3.39(1 \mathrm{H}, \mathrm{dd}, \mathrm{CH}, J 7.1$ and 5.4), $3.55(1 \mathrm{H}, \mathrm{d}, \mathrm{CH}, J$ 9.1), $3.66(1 \mathrm{H}, \mathrm{d}, \mathrm{CH}, J$ 9.1), $4.20(1 \mathrm{H}, \mathrm{dd}, \mathrm{CH}, J 8.5$ and 7.1$) ;{ }^{13} \mathrm{C}-\mathrm{NMR} \delta\left(75 \mathrm{MHz}, \mathrm{CDCl}_{3}\right) 14.6\left(\mathrm{CH}_{3}\right), 19.8\left(\mathrm{CH}_{3}\right)$, $44.5(\mathrm{CH}), 74.9\left(\mathrm{CH}_{2}\right), 78.3(\mathrm{C}), 81.4\left(\mathrm{CH}_{2}\right) ; \mathrm{m} / \mathrm{z}(\mathrm{EI}) 116\left(\mathrm{M}^{+}, 2 \%\right), 101(3), 84$ (6), 75 (88), 71 (80), 57 (15), 43 (100).

3-Hydroxy-3-methyloxane (6d). ${ }^{11}$ Eluent hexane-ethyl acetate 1:1 (Found: $\mathrm{M}^{+}, 116.0851$. $\mathrm{C}_{6} \mathrm{H}_{12} \mathrm{O}_{2}$ requires 116.0837); ${ }^{1} \mathrm{H}-\mathrm{NMR} \delta\left(300 \mathrm{MHz}, \mathrm{CDCl}_{3}\right) 1.08\left(3 \mathrm{H}, \mathrm{s}, \mathrm{CH}_{3}\right), 1.41-1.52(2 \mathrm{H}, \mathrm{m}$, $\left.\mathrm{CH}_{2}\right), 1.61-1.70(1 \mathrm{H}, \mathrm{m}, \mathrm{CH}), 1.74-1.88(1 \mathrm{H}, \mathrm{m}, \mathrm{CH}), 2.60(1 \mathrm{H}, \mathrm{s}, \mathrm{OH}), 3.25(1 \mathrm{H}, \mathrm{d}, \mathrm{CH}, J$ 11.4), $3.34(1 \mathrm{H}, \mathrm{dt}, \mathrm{CH}, J 11.1$ and 2.7$), 3.70(1 \mathrm{H}, \mathrm{dd}, \mathrm{CH}, J 11.4$ and 2.3), $3.78(1 \mathrm{H}, \mathrm{dt}, \mathrm{CH}, J$ 11.1 and 3.3); ${ }^{13} \mathrm{C}-\mathrm{NMR} \delta\left(75 \mathrm{MHz}, \mathrm{CDCl}_{3}\right) 22.4\left(\mathrm{CH}_{3}\right), 24.9\left(\mathrm{CH}_{2}\right), 35.8\left(\mathrm{CH}_{2}\right), 67.1(\mathrm{C}), 67.9$ $\left(\mathrm{CH}_{2}\right), 76.9\left(\mathrm{CH}_{2}\right) ; \mathrm{m} / \mathrm{z}(\mathrm{EI}) 116\left(\mathrm{M}^{+}, 21 \%\right), 84$ (33), 74 (17), 71 (72), ,58 (93), 43 (100).

3-Phenyl-3-hydroxy-2,3-dihydrobenzofuran (6g). Eluent hexane-ethyl acetate 10:1 (Found: $\mathrm{M}^{+}, 212.0838 . \mathrm{C}_{14} \mathrm{H}_{12} \mathrm{O}_{2}$ requires 212.0837); ${ }^{1} \mathrm{H}-\mathrm{NMR} \delta\left(300 \mathrm{MHz}, \mathrm{CDCl}_{3}\right) 2.36(1 \mathrm{H}, \mathrm{s}, \mathrm{OH})$, 4.49 (1H, d, CH, J 10.3), $4.68(1 \mathrm{H}, \mathrm{d}, \mathrm{CH}, J 10.3), 6.93-7.50(9 \mathrm{H}, \mathrm{m}, \mathrm{Ar}) ;{ }^{13} \mathrm{C}-\mathrm{NMR} \delta(75 \mathrm{MHz}$, $\left.\mathrm{CDCl}_{3}\right) 82.5(\mathrm{C}), 86.1\left(\mathrm{CH}_{2}\right), 110.7(\mathrm{CH}), 121.4(\mathrm{CH}), 124.4(\mathrm{CH}), 126.0(2 \times \mathrm{CH}), 127.5(\mathrm{CH})$, $128.2(2 \times \mathrm{CH}), 130.6(\mathrm{CH}), 132.2(\mathrm{C}), 142.6(\mathrm{C}), 160.6(\mathrm{C}) ; \mathrm{m} / \mathrm{z}(\mathrm{EI}) 212\left(\mathrm{M}^{+}, 100 \%\right), 195$ (48), 194 (53), 181 (29), 165 (43), 152 (26), 135 (48), 121 (39), 105 (25), 77 (41), 51 (17).

6,11-Dihydrobenzo[b,e]oxepin-11-on (7). ${ }^{12,13}$ Eluent hexane-ethyl acetate 10:1(Found: $\mathrm{M}^{+}$, 210.0682. $\mathrm{C}_{14} \mathrm{H}_{10} \mathrm{O}_{2}$ requires 210.0681); ${ }^{1} \mathrm{H}-\mathrm{NMR} \delta_{\mathrm{H}}\left(300 \mathrm{MHz}, \mathrm{CDCl}_{3}\right) 5.16\left(2 \mathrm{H}, \mathrm{s}, \mathrm{CH}_{2}\right), 7.01$ - 7.09 (2H, m, Ar), 7.33 (1H, d, Ar, J 7.3), 7.43 - 7.52(3H, m, Ar), 7.88 (1H, d, Ar, J 7.3), 8.21 $8.24(1 \mathrm{H}, \mathrm{m}, \mathrm{Ar}) ;{ }^{13} \mathrm{C}-\mathrm{NMR} \delta\left(75 \mathrm{MHz}, \mathrm{CDCl}_{3}\right) 73.5\left(\mathrm{CH}_{2}\right), 120.6(\mathrm{CH}), 122.0(\mathrm{CH}), 125.3(\mathrm{C})$, $127.7(\mathrm{CH}), 129.1(\mathrm{CH}), 129.4(\mathrm{CH}), 131.9(\mathrm{CH}), 132.6(\mathrm{CH}), 135.2(\mathrm{CH}), 135.6(\mathrm{CH}), 140.5$ (CH), 161.2 (C), 191.0 (CO); m/z (EI) $210\left(\mathrm{M}^{+}, 100 \%\right), 181$ (61), 153 (16), 152 (20), 89 (11), $76(9), 63(12)$.

\section{References}

1. Muffler, H.; Siegmund, G., Schwertfeger, W. J. Fluorine Chem. 1982, 21, 107.

2. (a) Benneche, T.; Undheim, K. Acta Chem. Scand. 1983, Ser. B 37, 93. (b) Benneche, T.; Strande, P.; Undheim, K. Synthesis 1983, 762. (c) Antonsen, Ø.; Benneche, T.; Undheim, K. Acta Chem. Scand. 1989, 43, 56. 
3. (a) Kagan, H.B.; Namy, J. Tetrahedron 1986, 42, 6573. (b) Sasaki,M.; Collin, J.; Kagan, H. B. New. J. Chem. 1992, 16, 89. (c) Imamoto, T.; Hatajima, T.; Takiyama, N.; Takeyama, T.; Kamiya, Y.; Yoshizawa, T. J. Chem. Soc., Perkin Trans. 1 1991, 3127. (d) Imamoto, T.; Takeyama, T.; Yokoyama, M. Tetrahedron Lett. 1984, 25, 3225. (e) White, J. D.; Somers, T. C. J. Am. Chem. Soc. 1987, 109, 4424. (f) Antonsen, Ø.; Benneche, T.; Undheim, K. Acta Chem. Scand. 1992, 46, 757.

4. (a) Keay, B. A.; Dibble, P. W. In Comprehensive Heterocyclic Chemistry II, C. W. Bird Ed., Pergamon: Oxford, 1996, Vol. 2, p 395. (b) Green, G. R.; Evans, J. M.; Vong, A. K. In Comprehensive Heterocyclic Chemistry II, C. W. Bird Ed., Pergamon: Oxford, 1996, Vol. 5, p. 469.

5. (a) Corey, E. J.; Bock, M. C. Tetrahedron Lett. 1975, 38, 3269. (b) Antonsen, Ø.; Benneche, T.; Undheim, K. Acta Chem. Scand. 1988, Ser. B 42, 515.

6. Pojer, P. M.; Angyal, S. J. Tetrahedron Lett. 1976, 35, 3067.

7. Nordlien, S.; and Benneche, T. MD thesis, University of Oslo, 1998.

8. Ringom, R.; and Benneche, T. Acta Chem. Scand. 1999, 53, 41.

9. Fukuzawa, S.-I.; Furuya, H.; Tsuchimoto, T. Tetrahedron 1996, 52, 1953.

10. Omichi, H.; Machida, H.; Miyakoshi, T.; Saito, S. Nippon Kagaku Kaishi 1977, 7, 1021.

11. Budzikiewicz, H.; and Grotjahn, L. Tetrahedron 1972, 28, 1881.

12. Kurokaawa, M.; Sato, F.; Masuda, Y.; Yoshida, T.; Ochi, Y.; Zushi, K.; Fujiwara, I.; Naruto, S.; Uno, H.; Matsumoto, J.-I. Chem. Pharm. Bull. 1991, 39, 2564.

13. Stach, K.; Springler, H. Monatsh. Chem. 1962, 93, 890. 